\title{
Ultrafast electron transfer, recombination and spin dynamics
}

\author{
P. Gilch a , F. Pöllinger-Dammer a , U.E. Steiner ${ }^{\text {b }}$, M.E. Michel-Beyerle ${ }^{\text {a }}$ \\ ${ }^{a}$ Institut fïr Physikalische und Theoretische Chemie, Technische Universität München, D-85748 Garching, Germany \\ ${ }^{b}$ Fakultät für Chemie, Universität Konstanz, D-78434 Konstanz, Germany
}

\begin{abstract}
The quenching of excited singlet methylene blue $\left({ }^{1} \mathrm{MB}^{+*}\right)$ by $\mathrm{N}, \mathrm{N}$-dimethylaminomethylferrocene $(\mathrm{FcN})$ in acetonitrile at room temperature has been studied using femtosecond pump-probe absorption spectroscopy. At high $\mathrm{FcN}$ concentration static quenching via an intermolecular electron transfer mechanism constitutes the predominating decay channel for ${ }^{1} \mathrm{MB}^{+*}$. The time constants of the large amplitude components for the forward electron transfer from $\mathrm{FcN}$ donor to ${ }^{1} \mathrm{MB}^{+*}$ and the subsequent recombination process recovering the ground states have been determined to be $390 \mathrm{fs}$ and $1 \mathrm{ps,}$, respectively. Thus, the majority of radical pairs has recombined before paramagnetic relaxation induces spin mixing giving rise to triplet-phased radical pairs. These can escape geminate recombination as the system does not offer low-lying local triplet states to be occupied in the recombination process.
\end{abstract}

\section{Introduction}

Recently, it has been shown that spin dynamics on the picosecond timescale manifest themselves in the chemical reaction kinetics of electron donor/acceptor (D/A) systems involving paramagnetic transition metal complexes. In such systems, which can be characterized as radical pairs (RP) involving the paramagnetic complex as a d-electron radical moiety, high magnetic fields in the range of several tesla are needed to affect the spin motion and thereby, indirectly, reaction rates and yields of electron transfer (ET) reactions [1]. Such a mechanism is closely related to the low field effects which are based on the classical radical pair mechanism and have been well explored with typical organic radicals applying the full arsenal of spin chemical techniques such as MARY [2], RYDMR [3], CIDNP and CIDEP spectroscopy [4]. Whereas, however, in these classical cases the weak hyperfine coupling is the relevant interaction leading to spin evolution on the timescale of nanoseconds (i.e. at the limit of the time resolution of ESR) and to magnetic saturation fields of the order of several 100 gauss, the interactions that matter in the case of D/A systems with the above mentioned paramagnetic complexes are due to spin-orbit coupling and the spin chemical effects require saturation fields up to $10 \mathrm{~T}$. Thus, in terms of the magnitude of the field scale as well as the shortness of the timescale the latter systems exceed the limits of classical spin chemistry by three orders of magnitude.

So far, metal complexes of the iron group have figured most prominently in the new spin chemical area dealing with strongly spin-orbit coupled metal containing D/A systems. In the formation of the RP state the metal complex has either been photoexcited or been in its ground state and has acted as an electron donor or acceptor. Examples of the photoexcited donor case are provided by $\mathrm{Ru}$-trisbipyridine 
type complexes [1], an example of a ground state acceptor case has been reported with the $\mathrm{Ni}^{2+}$-transmetallated analogue of bacteriochlorophyll in the photosynthetic reaction center [5]. The most recent example in this class of effects is the case of methylene blue $\left(\mathrm{MB}^{+}\right)$triplet quenching by ferrocene $(\mathrm{Fc})$ [6] where the metal complex acts as a ground state electron donor. The reaction scheme and energy scale of this reaction is shown in Fig. 1. In solutions of low concentration $\left(\leqslant 10^{-4} \mathrm{M}\right)$ of $\mathrm{Fc}$ the first excited singlet state of $\mathrm{MB}^{+}$decays intramolecularly with a time constant of $\approx 1 \mathrm{~ns}[7,8]$ yielding the triplet state ${ }^{3} \mathrm{MB}^{+*}$ with a quantum yield of 0.5 (value in ethanol [9]). As described by Tamura et al. [10] $\mathrm{Fc}$ quenches ${ }^{3} \mathrm{MB}^{+*}$ by electron transfer at a close to diffusion-controlled rate (rate constant $k_{\mathrm{qt}}$ ) whereby free ferrocenium cations $\mathrm{Fc}^{+}$and semi-reduced MB radicals are formed with an efficiency of about 0.2 . The fact that this efficiency is clearly smaller than 1 has been assigned to the successful competition of fast geminate recombination (REC), i.e. backward ET (rate constant $k_{\text {rec }}$ ), with diffusional separation (rate constant $k_{\text {ce }}$ ) of the geminate $\mathrm{RP}$, i.e. the efficiency of radical formation is to be interpreted as the efficiency of cage escape of the geminate RP.

Since, on quenching of ${ }^{3} \mathrm{MB}^{+*}$, the RP is created with initial triplet spin the REC process regenerating the singlet ground state reactants requires a fast $\mathrm{T} \rightleftharpoons \mathrm{S}$ spin conversion. The role of spin multiplicity and spin relaxation in this mechanism has been

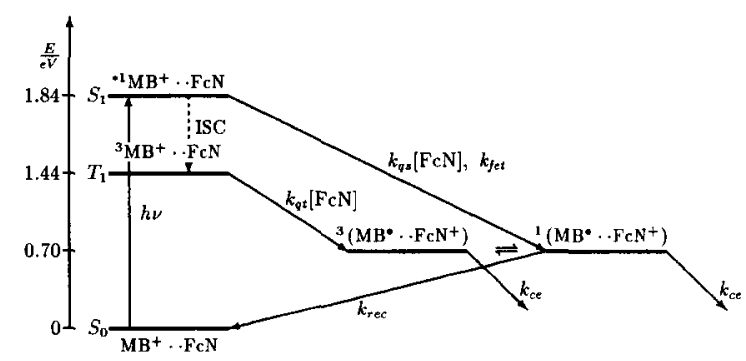

Fig. 1. Simplified reaction scheme describing the relevant kinetic and energetic parameters of the $\mathrm{MB}^{+} / \mathrm{FcN}$ couple. $k_{\mathrm{qs}}$ and $k_{\mathrm{qt}}$ are the quenching constants of ${ }^{1} \mathrm{MB}^{+*}$ and ${ }^{3} \mathrm{MB}^{+}$by $\mathrm{FcN}$ respectively, $k_{\text {fet }}$ and $k_{\text {rec }}$ are the rate constants for the forward transfer and the recombination in the limit of high $\mathrm{FcN}$ concentrations, $k_{\mathrm{ce}}$ is the cage escape rate constant for the radical pair and $\rightleftharpoons$ symbolizes singlet-triplet mixing. studied in detail by the spin chemical investigation described in Ref. [6] wherein the magnetic field dependence of the cage escape efficiency was determined. The magnetic field decreases the yield of free radicals which was explained as a result of magnetic-field-induced triplet-singlet mixing in the RP, supporting the singlet selective REC process. From a simulation of the experimental field dependence on the basis of this spin chemical mechanism the rate constants of the mechanism have been quantitatively deduced, yielding $1.6 \mathrm{ps}$ as the time constant for the spin-allowed backward ET process and 6 ps for the spin relaxation in the ferrocenium cation which corresponds to a value of $0.04 \mathrm{ps}^{-1}$ for the rate constant of $\mathrm{T} \rightleftharpoons \mathrm{S}$ or $0.12 \mathrm{ps}^{-1}$ for the rate constant of $S \rightleftharpoons T$ conversion. Although these parameters have been determined in a consistent manner, the spin chemical way of assessing them is based on time integrated, indirect measurements and it must be considered an experimental challenge to provide direct time resolved access to the underlying elementary processes.

In the present work, femtosecond time-resolved spectroscopy has been applied to investigate the processes of electron transfer and RP recombination in the $\mathrm{MB}^{+} / \mathrm{Fc}$ system in solutions of high ferrocene concentration so that practically no diffusion was necessary to create RPs in the forward electron transfer reaction and the REC process of geminate RPs could be followed with maximum time-resolution. Our results provide direct evidence for the rate processes deduced from the spin chemical study.

\section{Materials and experimental methods}

\subsection{Materials}

Methylene blue (Fluka), ferrocene (Aldrich), dimethylaminomethylferrocene (Aldrich) and acetonitrile (UVASOL, Merck) were used as supplied. Sample solutions were freshly prepared prior to each measurement. Glass cuvettes with $1 \mathrm{~mm}$ optical path length were used for all experiments.

Measurements were performed with a derivative of ferrocene: N,N-dimethylaminoferrocene $(\mathrm{Fc}-$ $\mathrm{CH}_{2}-\mathrm{NMe}_{2}, \mathrm{FcN}$ ), because for singlet exited state 
quenching experiments quencher concentrations of $\geqslant 0.1 \mathrm{M}$ are necessary and the saturation concentration of ferrocene in acetonitrile is $0.14 \mathrm{M}$ [11]. $\mathrm{FcN}$ is liquid at room temperature and highly soluble in acetonitrile. Its UV/VIS spectrum is similar to that of $\mathrm{Fc}$ [12]. The reduction potential of $\mathrm{FcN}^{+}$in water is $423 \mathrm{mV}$ (SHE) [13] compared to $400 \mathrm{mV}$ for $\mathrm{Fc}^{+}$ in water (SHE) [14]. It seems unlikely that the difference in the potentials is much larger in acetonitrile, for which no published reduction potential of $\mathrm{FcN}^{+}$is available. Because of the small difference in reduction potential, we expect similar ET behaviour for the two compounds. In fact, for the lowest quencher concentration $(0.1 \mathrm{M})$ the employed pump-probe measurements performed on $\mathrm{MB}^{+} / \mathrm{Fc}$ and $\mathrm{MB}^{+} / \mathrm{FcN}$ yielded virtually identical results.

\subsection{Apparatus}

Time-resolved transient absorption measurements were performed using pump-probe apparatus with a time resolution better than $200 \mathrm{fs}$ and a repetition rate of $1 \mathrm{kHz}$. To generate excitation pulses at 650 $\mathrm{nm}$, the output of a Ti-sapphire laser system (COHERENT / BMI), described in detail in refs. [15] and [16], was frequency doubled and used to pump an optical parametric generator, followed by a twostep optical parametric amplifier system (OPG/OPA, BMI VENTURI II) [17]. With this apparatus we were able to produce $150 \mathrm{fs}$ pulses at $650 \mathrm{~nm}$ with 5 $\mu \mathrm{J}$ pulse energy and a pulse to pulse fluctuation of less than $20 \%$ rms. Probe pulses were generated by selecting a $15 \mathrm{~nm}$ wide portion out of a white light continuum generated by focusing 1-2 $\mu \mathrm{J}$ pulses at $780 \mathrm{~nm}$ in an ethanol flow-through cell. Pump and probe pulses were focused on the sample (pump beam diameter $800 \mu \mathrm{m}$, probe beam diameter 300 $\mu \mathrm{m})$ under a small angle. The probe polarization was set to magic angle conditions to avoid rotational depolarization effects.

Transient absorbance was calculated from two consecutive absorption measurements using a 500 $\mathrm{Hz}$ chopper to block every second excitation pulse. Each transient absorbance value was then normalized to the excitation energy to eliminate the effects of fluctuating excitation energy. Transient absorbance values down to $0.2 \mathrm{mOD}$ were detectable after averaging over 8000 normalized measurements. The time resolution of our pump-probe apparatus was determined to be $180 \mathrm{fs}$ by Gaussian deconvolution of the signal rise in systems yielding transients with long lifetimes. An example is provided by the signals obtained with $\mathrm{MB}^{+}$solutions at a probe wavelength of $630 \mathrm{~nm}$.

\subsection{Analysis of time resolved measurements}

The D/A system under study here is a bimolecular one. Consequently, we are dealing with a distribution of D/A distances and relative orientations. In addition, quenching may occur by quencher molecules, which are close to the excited molecule at the moment of excitation (static quenching) or by molecules diffusing towards the excited molecule (dynamic quenching). This complexity is expressed in an extremely non-exponential time dependence of the transient state populations. A thorough analysis of this time dependence is beyond the scope of this Letter (for a recent treatment of the problem see Ref. [18]). To get an idea of the timescales involved in the quenching process, the experimental data were fitted to a multi-exponential decay function convoluted with an instrument response function $B\left(t-t_{0}\right)$ centered at $t_{0}$ :

$$
\begin{aligned}
\Delta A(t)= & \int_{0}^{+\infty}\left(\Delta A_{0}+\sum_{i=1}^{n} \Delta A_{i} \exp \left(-\frac{t^{\prime}}{\tau_{i}}\right)\right) \\
& \times B\left(t-t^{\prime}-t_{0}\right) \mathrm{d} t^{\prime},
\end{aligned}
$$

where $\Delta A(t)$ is the difference absorbance at time $t$, $\Delta A_{i}$ is the amplitude of the component with lifetime $\tau_{i}$ and $\Delta A_{0}$ is the offset due to long-lived ( $>1 \mathrm{ns)}$ species (e.g. triplet states). The instrument response function $B\left(t-t_{0}\right)$ was modeled by a Gaussian with a FWHM of $\approx 180 \mathrm{fs}$ corresponding to the cross correlation of pump and probe pulses. This cross correlation was experimentally verified by the deconvolution procedure specified in Section 2.2.

\section{Results and discussion}

\subsection{Femtosecond pump-probe measurements}

For all the femtosecond pump-probe measurements the excitation was tuned to $650 \mathrm{~nm}$, corre- 


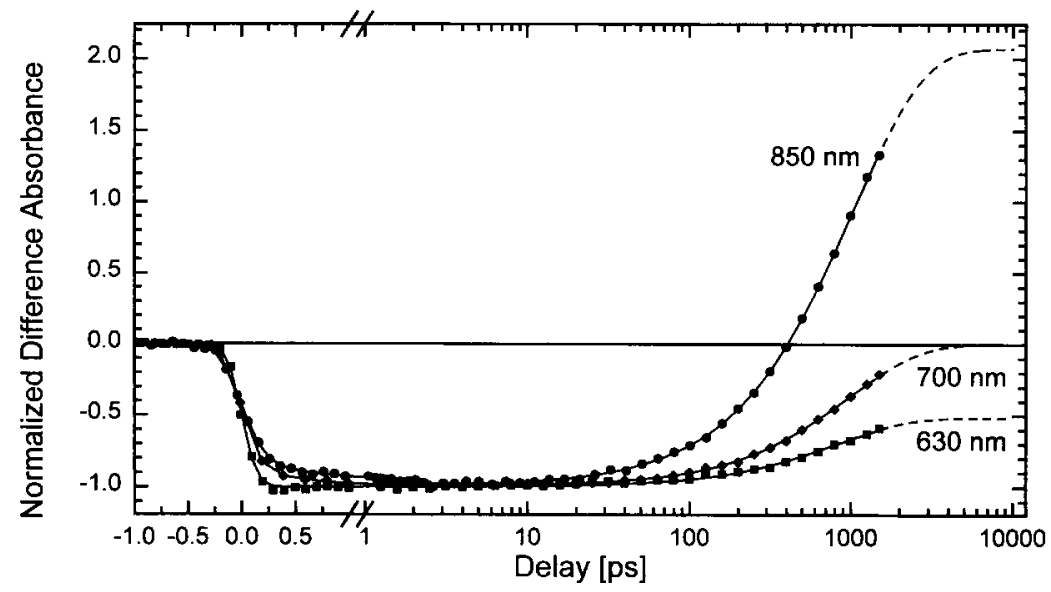

Fig. 2. Pump-probe measurements on pure $\mathrm{MB}^{+}$in acetonitrile. The timescale is linear from -1 to 1 ps and logarithmic from there on. For the sake of comparison the measurements at different probe wavelengths are normalized with respect to each other. The actual values of the difference absorbances (at several ps delay time) were $-75,-45$ and -4 mOD for 630,700 and 850 nm, respectively. The solid lines describe the best fits according to Eq. (1), the dashed lines are extrapolations of these functions.

sponding to the maximum of the $\mathrm{MB}^{+} \mathrm{S}_{0} \rightarrow \mathrm{S}_{1}$ absorption. The extinction coefficient $\varepsilon_{650 \mathrm{~nm}}$ of $\mathrm{MB}^{+}$ is around $90000 \mathrm{M}^{-1} \mathrm{~cm}^{-1}$ [7]. The concentration of $\mathrm{MB}^{+}$was $0.17 \mathrm{mM}$ yielding an absorbance of 1.6 at $650 \mathrm{~nm}$ in a $1 \mathrm{~mm}$ cuvette. Ferrocene [12] and dimethylaminomethylferrocene (own measurements) have extinction coefficients of $\approx 1 \mathrm{M}^{-1} \mathrm{~cm}^{-1}$ in this spectral region. Therefore, even for the highest $\mathrm{FcN}$ concentration applied the portion of light absorbed by $\mathrm{FcN}$ is less than $15 \%$.

Methylene blue, its lowest excited states ${ }^{1} \mathrm{MB}^{+*}$ $[19],{ }^{3} \mathrm{MB}^{+*}[10]$ and its semi-reduced form $\mathrm{MB}$. [10] have much higher extinction coefficients in the visible region than ferrocene [12] and its cation [20]. Consequently, the spectral changes observed after excitation are essentially due to the various states of methylene blue. We chose three probe wavelengths characterising these different states: $630 \mathrm{~nm}$, absorption of $\mathrm{MB}^{+}$, negative difference absorbance due to ground state depletion; $700 \mathrm{~nm}$, stimulated emission of singlet state ${ }^{\mathrm{I}} \mathrm{MB}^{+*}$; and $850 \mathrm{~nm}$, red wing of the ${ }^{\prime} \mathrm{MB}^{+*}$ stimulated emission and absorption of the triplet state ${ }^{3} \mathrm{MB}^{+*}[21]$.

Monitoring the radical form of methylene blue $\mathrm{MB}^{-}$is not possible under our experimental conditions, as its maximum absorption in the visible region lies around $430 \mathrm{~nm}$ [21] where the samples are opaque due to ferrocene absorption $\left(\lambda_{\max }=440 \mathrm{~nm}\right)$.

\subsubsection{Excited state kinetics of ${ }^{I} M B^{+*}$ in the $a b$ - sence of $F C N$}

The behaviour of the first excited singlet state ${ }^{\mathrm{I}} \mathrm{MB}^{+*}$ is monitored by the measurement at $700 \mathrm{~nm}$ (Fig. 2). The negative difference absorbance resulting from stimulated emission shows a rise time component of $440 \mathrm{fs}$ and a characteristic decay time of $1 \mathrm{~ns}$. No offset was necessary to fit the data. The rise time component could be due to a dynamic Stokes shift caused by dynamic solvation. This assumption is supported by the measurements of Horng et al. [22]. For coumarin 153 in acetonitrile time constants of 90 and $630 \mathrm{fs}$ for the temporal behaviour of the spectral response function were reported which reflect the dynamic response of the solvent. The latter value compares well with the 440 fs we measured ', the shorter time constant is below our instrument response function and is therefore not

\footnotetext{
' It should be kept in mind that the rise time we measure and the time dependence of the spectral response function are not directly comparable. The spectral response function describes the shift in the maximum of the spectrum during solvation. The time dependence monitored at a fixed wavelength, on the other hand, not only reflects this shift but also the curvature of the part of the emission spectrum passing by the monitoring wavelength. Therefore, probing at different wavelengths leads to slightly different time dependent signals.
} 


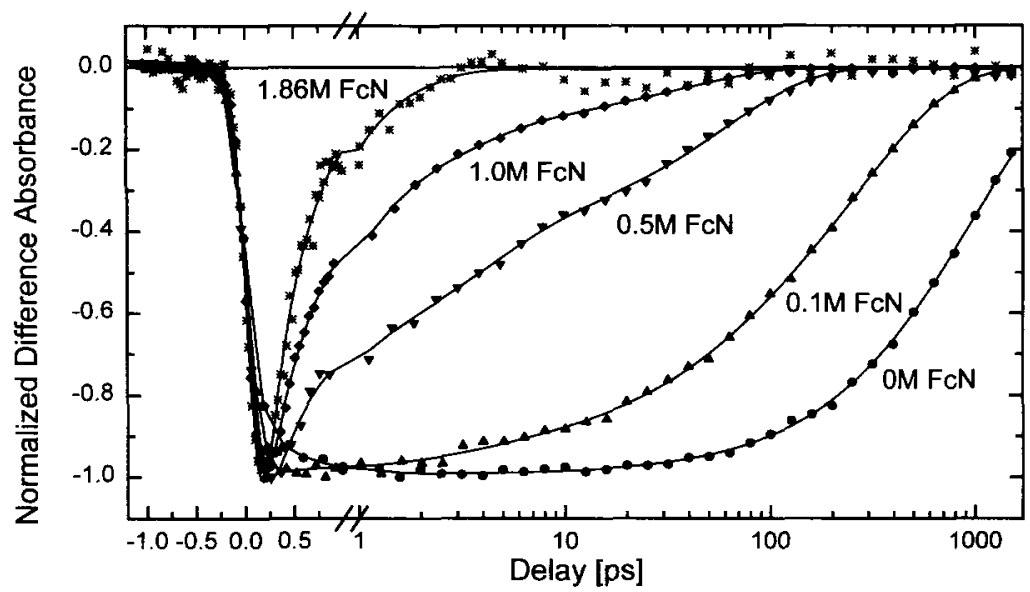

Fig. 3. Pump-probe measurements of $\mathrm{MB}^{+}$in the presence of different concentrations of FcN. Probe wavelength tuned to $700 \mathrm{~nm}$, monitoring the decay of the ${ }^{1} \mathrm{MB}^{+*}$ state.

resolved. The 1 ns time constant is characteristic of the decay of the ${ }^{1} \mathrm{MB}^{+*}$ state and comparable to the reported lifetimes of 1.1 [8] and $0.75 \mathrm{~ns}$ [7].

For the probe beam tuned to $630 \mathrm{~nm}$ (Fig. 2) a negative difference absorbance is seen due to bleaching of the ground state. These data can be fitted using only one time constant and allowing for an offset. No fast rise component is needed to fit the data. This is in accordance with the assumption that the rise in the difference absorbance in the measurement at $700 \mathrm{~nm}$ is due to dynamic solvation of the excited singlet state which is not seen in the bleach- ing of the ground state absorption. Recovery of the ground state absorption occurs with a time constant of $0.8 \mathrm{~ns}$, comparable to the depletion kinetics of the ${ }^{1} \mathrm{MB}^{+*}$ state. The time constants in the nanosecond region cannot be determined precisely as our delay line is restricted to times below $1.5 \mathrm{~ns}$. The ground state is recovered by $\approx 50 \%$ in agreement with the reported triplet quantum yield of 0.52 (in ethanol [9]) of ${ }^{1} \mathrm{MB}^{+*}$.

Formation of the triplet state ${ }^{3} \mathrm{MB}^{+*}$ is observed when probing at $850 \mathrm{~nm}$ (Fig. 2). Immediately after excitation the difference absorbance is negative be-

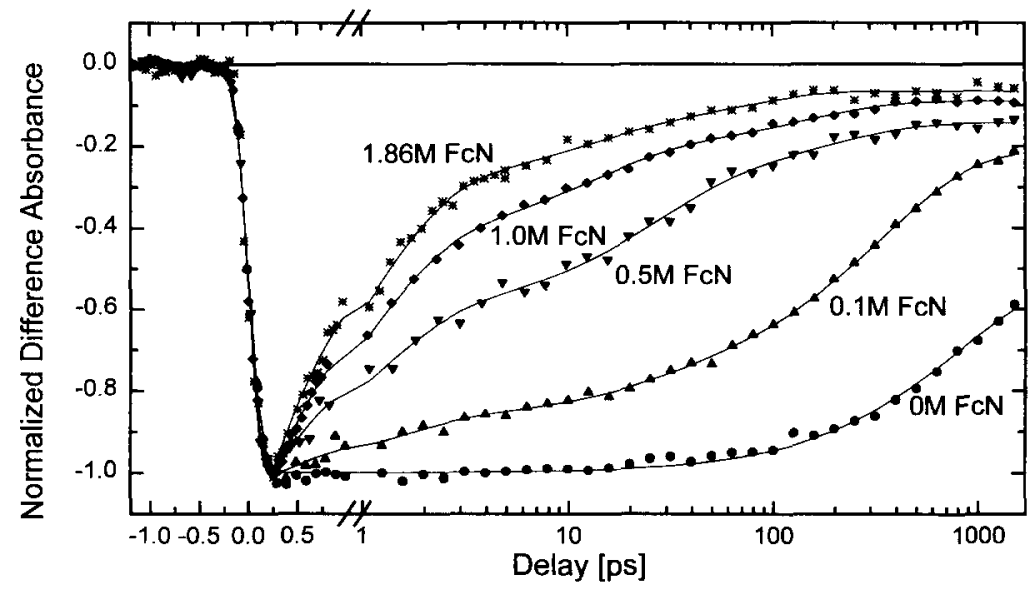

Fig. 4. Pump-probe measurements of $\mathrm{MB}^{+}$in the presence of different concentrations of $\mathrm{FcN}$. Probe wavelength tuned to $630 \mathrm{~nm}$, monitoring the recovery of the $\mathrm{MB}^{+}$ground state. 
cause of the red wing of the stimulated emission. Again, a rise is seen with a characteristic time of 640 fs, i.e. somewhat longer than in the measurement at $700 \mathrm{~nm}$. This rise is again caused by the dynamic solvation process discussed above. The negative signal decays with a time constant of $1 \mathrm{~ns}$, comparable to the constants determined at the other probe wavelengths, resulting in a positive difference absorbance due to the population of ${ }^{3} \mathrm{MB}^{+*}$.

\subsubsection{Excited state kinetics of ${ }^{\prime} \mathrm{MB}^{+*}$ in presence of $F c N$}

As expected, the addition of $\mathrm{FcN}$ reduces the lifetime of ${ }^{1} \mathrm{MB}^{+*}$ (Figs. 3-5). For the lowest concentration of $\mathrm{FcN} 0.1 \mathrm{M}$ the decay kinetics are multi-exponential and fitted by three exponentials to parameterize the distribution of lifetimes. For all probe wavelengths the time constant of the major amplitude is $\approx 300 \mathrm{ps}$. If we assume that this contribution is dominated by dynamic quenching, the quenching constant $k_{\mathrm{qs}}$ is given by

$k=k_{0}+k_{\mathrm{qs}}[\mathrm{FcN}]$,

where $k_{0}$ is the intrinsic decay rate constant of ${ }^{1} \mathrm{MB}^{+*}$, as determined in the absence of $\mathrm{FcN}$, and $k$ is the constant in the presence of $\mathrm{FcN}$. The resulting quenching constant $k_{\mathrm{qs}}=2.3 \times 10^{10} \mathrm{M}^{-1} \mathrm{~s}^{-1} \mathrm{com}-$ pares with a triplet quenching constant of $1.1 \times 10^{10}$ $\mathrm{M}^{-1} \mathrm{~s}^{-1}$ [10]. The order of magnitude of both constants supports the notion that in both cases the quenching is diffusion-controlled. The population of the local triplet state ${ }^{3} \mathrm{MB}^{+*}$ as seen in the residual ground state bleaching in Fig. 4 and in the long-lived positive difference absorbance in Fig. 5 should be reduced by the same factor as the ${ }^{1} \mathrm{MB}^{+*}$ lifetime. Indeed, this is the case with both values reduced by a factor of $\approx 3$ upon addition of $0.1 \mathrm{M} \mathrm{FcN}$. The decay of the triplet state indicated by the data points collected for times later than $1 \mathrm{~ns}$ reflects the quenching of the ${ }^{3} \mathrm{MB}^{+*}$ state by $\mathrm{FcN}$ already studied in detail by Tamura et al. [10].

Increasing the concentration of $\mathrm{FcN}$ shows switching from dynamic to static quenching. Probing the ${ }^{1} \mathrm{MB}^{+*}$ stimulated emission at $700 \mathrm{~nm}$ (Fig. 3), the longest lifetime $\tau_{3}$ in Table 1 decreases in a way predicted by Eq. (2), i.e. inversely proportional to the $\mathrm{FcN}$ concentration. The amplitude of this dynamic contribution decreases steadily and vanishes for the highest concentrations of $\mathrm{FcN}$. Parallel to this decrease the amplitude of an ultrashort component $\tau_{1}$ of $\approx 390 \mathrm{fs}$ increases, the time constant being essentially concentration independent. The intermediate time constant $\tau_{2}$ decreases with increasing $\mathrm{FcN}$ concentration, as does its amplitude. We assign the two short time constants $\tau_{1}$ and $\tau_{2}$ to static quenching.

The ground state recovery measurement (Fig. 4) also shows a short component for all $\mathrm{FcN}$ concentrations with a characteristic time of $\approx 1$ ps which is significantly longer than the corresponding major

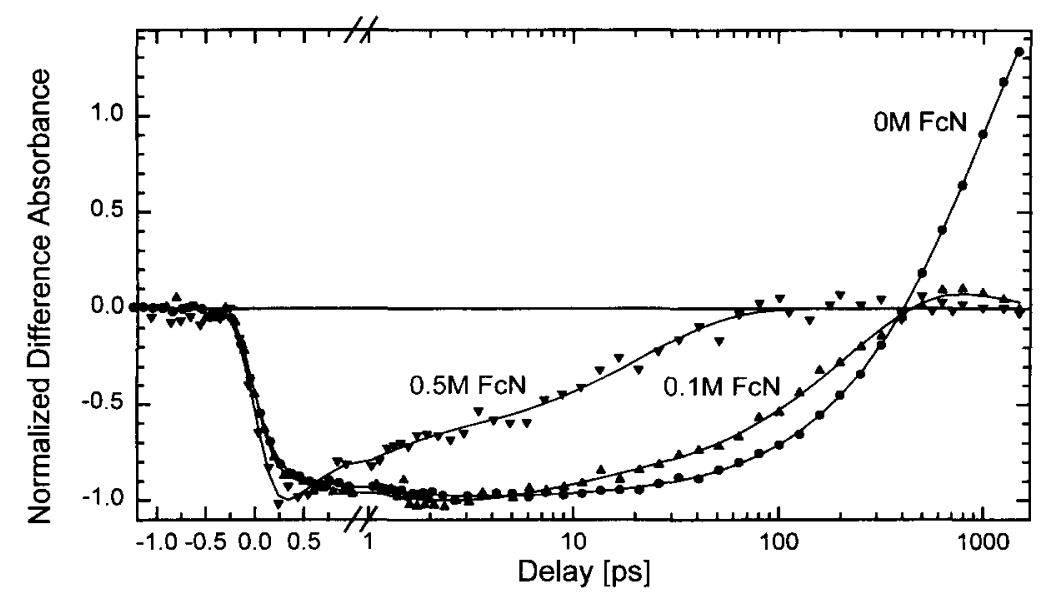

Fig. 5. Pump-probe measurements of $\mathrm{MB}^{+}$in the presence of different concentrations of $\mathrm{FcN}$. Probe wavelength tuned to $850 \mathrm{~nm}$, monitoring the decay of the ${ }^{1} \mathrm{MB}^{+*}$ state and the formation of the triplet absorption. 
Table 1

Numerical parameters of the fit functions displayed as solid lines in Figs. 2-5

\begin{tabular}{lllllllllll}
\hline$\lambda_{\text {probe }}$ & $c$ & $\Delta A_{1}$ & $\tau_{1}$ & $\Delta A_{2}$ & $\tau_{2}$ & $\Delta A_{3}$ & $\tau_{3}$ & $\Delta A_{4}$ & $\tau_{4}$ & $\Delta A_{0}$ \\
\hline 700 & 0 & 0.153 & 0.44 & - & - & -1.0 & 989 & - & - & - \\
& 0.1 & - & - & -0.166 & 18.1 & -0.834 & 269 & - & - & - \\
& 0.5 & -0.435 & 0.38 & -0.260 & 3.55 & -0.305 & 59.3 & - & - & - \\
& 1.0 & -0.707 & 0.47 & -0.189 & 2.61 & -0.104 & 35.9 & - & - & - \\
& 1.86 & -0.785 & 0.33 & -0.215 & 0.98 & - & - & - & - & - \\
630 & 0 & - & - & - & - & -0.476 & 821 & - & - & -0.524 \\
& 0.1 & -0.162 & 1.14 & -0.096 & 27.1 & -0.537 & 353 & - & - & -0.205 \\
& 0.5 & -0.447 & 1.00 & -0.289 & 25.1 & -0.137 & 183 & - & - & -0.128 \\
& 1.0 & -0.623 & 0.93 & -0.194 & 12.0 & -0.109 & 145 & - & - & -0.075 \\
& 1.86 & -0.738 & 0.92 & -0.110 & 9.20 & -0.099 & 60 & - & - & -0.054 \\
850 & 0 & 0.183 & 0.84 & - & - & -3.06 & 1038 & - & - & 2.06 \\
& 0.1 & 0.264 & 1.04 & -0.165 & 6.95 & -1.752 & 271 & 0.830 & $500(\mathrm{f})$ & - \\
& 0.5 & -0.430 & 0.69 & -0.570 & 21.1 & - & - & - & - & - \\
\hline
\end{tabular}

$\lambda_{\text {probe }}$ is the probe wavelength in $\mathrm{nm}, \Delta A_{1,2,3,4}$ are the relative amplitudes belonging to the time constants $\tau_{1,2,3,4}$ in ps and $\Delta A_{0}$ is the offset due to long-lived species. Note that the sum of the negative amplitudes and the negative offset equals one for the 630 and $700 \mathrm{~nm}$ data. ' - ' indicates that the respective parameter was not needed to yield a satisfying fit of the data, and the parameters labeled (f) were held fixed during fitting.

short component of $390 \mathrm{fs}$ for the decay of ${ }^{1} \mathrm{MB}^{+*}$. This mismatch of time constants for the decay of the excited state and ground state recovery points to an intermediate state being formed during the decay. The amplitudes of both these components grow with increasing concentrations of $\mathrm{FcN}$ in nearly the same way.

Contrary to the $700 \mathrm{~nm}$ behaviour, the $630 \mathrm{~nm}$ trace for the highest quencher concentration shows components with time constants in the range 10-60 ps and an offset of 0.05 .

As demonstrated by the $850 \mathrm{~nm}$ time trace (Fig. 5) no triplet ${ }^{3} \mathrm{MB}^{+*}$ is produced for $\mathrm{FcN}$ concentrations of $0.5 \mathrm{M}$ indicating that all the ${ }^{1} \mathrm{MB}^{+*}$ decay is due to quenching. Therefore, for the higher $\mathrm{FcN}$ concentrations no measurements at this wavelength were undertaken.

In summary, in the presence of high $(\approx 1.9 \mathrm{M})$ concentrations of $\mathrm{FCN}$ the essential features of the $\mathrm{FcN} / \mathrm{MB}^{+}$system are the following ones.

(1) The majority of ${ }^{1} \mathrm{MB}^{+*}$ is quenched with a time constant of $390 \mathrm{fs}$ and the characteristic time of the major amplitude of the ground state recovery is 1 ps. The discrepancy in the time constants of the excited state decay and recovery of the ground state points to the population of an intermediate state and thereby rules out excited state energy transfer ${ }^{2}$. Energy transfer can also be excluded on the basis of trivial energetic reasons: the excited state energies of $\mathrm{Fc}$, which should be close to those of $\mathrm{FcN}$, are 2.81 $\mathrm{eV}$ for $S_{1}$ [23] and $2.4 \mathrm{eV}$ for $T_{1}$ [23] being well above that of ${ }^{1} \mathrm{MB}^{+*}(1.84 \mathrm{eV}$ [7]).

(2) While the decay of ${ }^{1} \mathrm{MB}^{+*}$ is fully completed in less than $5 \mathrm{ps}$, the recovery of the ground state $\mathrm{MB}^{+}$is not completed even within $1 \mathrm{~ns}$, although by that time the unrecombined fraction is less than 10 compared to the forward ET is indicative of $S \rightleftharpoons T$ spin dynamics reducing the initial singlet character of the RP and of cage escape yielding a certain amount of long-lived free radicals.

\subsection{Discussion of forward and reverse electron transfer processes}

\subsubsection{Fast components of FET and REC}

Since ns-time-resolved quenching experiments have shown that ET quenching is close to being

\footnotetext{
${ }^{2}$ The results of our measurements at $850 \mathrm{~nm}$ exclude an enhanced intersystem crossing to the ${ }^{3} \mathrm{MB}^{+*}$ state that might arise due to an external heavy atom effect of $\mathrm{FcN}$.
} 
diffusion controlled for the excited triplet state ${ }^{3} \mathrm{MB}^{+*}$, there is good reason to assume that it is diffusion controlled for the excited singlet state ${ }^{1} \mathrm{MB}^{+*}$ separated by an energy gap of only $0.4 \mathrm{eV}$ from the triplet state. We therefore propose an ET mechanism as illustrated in Fig. 1: FcN in its ground state donates an electron to singlet excited ${ }^{1} \mathrm{MB}^{+*}$, thereby producing a singlet-phased $\mathrm{RP} \mathrm{MB}^{\circ}$

$\cdots \mathrm{FcN}^{+}$. In the presence of the highest concentration of $\mathrm{FcN}$ the shortest time constant for this charge shift reaction is $390 \mathrm{fs}$. For such high concentrations the following simple estimate supports the assumption that each excited molecule has several donors in close proximity.

A donor concentration of $1.9 \mathrm{M}$ corresponding to a particle density of $10^{-3} \AA^{-3}$, i.e. one molecule in a cube of $(10 \AA)^{3}$, allows for an average center-tocenter distance between $\mathrm{FcN}$ and $\mathrm{MB}^{+}$of $\approx 7 \AA$. The 'radius' of $\mathrm{FcN}$ is $\approx 3 \AA$ (from crystallographic data [24] for $\mathrm{Fc}$ ) and the 'thickness' of the planar molecule $\mathrm{MB}^{+}$is $\approx 2 \AA$, which results in an average edge-to-edge distance of only $\approx 2 \AA$. ET therefore occurs under close contact conditions.

The FET in the close contact pairs (characterized by rate constant $k_{\text {fet }}$ in Fig. 1) cannot be rate determined by reorientational motion which is easily shown by estimating the rotational correlation time $\tau_{\mathrm{r}}$. The Debye relation

$\tau_{\mathrm{r}}=\frac{4 \pi \eta r^{3}}{3 k T}$

for this correlation time yields a value of $10 \mathrm{ps}$ using $0.341 \times 10^{-3}$ Pas [25] for the dynamic viscosity $\eta$ of acetonitrile at room temperature and $3 \AA$ for the hydrodynamic radius of $\mathrm{FcN}$ (the smaller of the two reactants) ${ }^{3}$. This correlation time is much longer than the shortest time constant of 390 fs observed for the ${ }^{1} \mathrm{MB}^{+*}$ depletion and, in the case of the highest concentrations of $\mathrm{FcN}$, even longer than the longest time constant of 0.98 ps.

\footnotetext{
${ }^{3}$ A pump-probe experiment with $\mathrm{MB}^{+}$, not taken under magic angle conditions of the excitation and probe polarization, gave an additional component of $\approx 40 \mathrm{ps}$, which reflects the rotational depolarization of ${ }^{1} \mathrm{MB}^{+*}$. This demonstrates that the magnitude of the calculated value is realistic keeping in mind that $\mathrm{MB}^{+}$is larger than $\mathrm{FcN}$.
}

The amplitude of this shortest component increases with growing $\mathrm{FcN}$ concentration because the portion of excited acceptors surrounded by quenchers in an optimal (or nearly optimal) orientation of FET increases. The $390 \mathrm{fs}$ component should therefore be the intrinsic forward transfer time constant $\tau_{\text {fet }}$ for a contact pair.

The shortest and major component in the ground state recovery exhibits a time constant of $1 \mathrm{ps}$. It is still too short for rotational diffusion to account for it. So this time constant should describe the REC of the RP, i.e. the backward ET of those pairs in optimal relative orientation for FET. Furthermore, it should be noted at this point, that this fast time constant of ground state recovery compares favorably with the $1.6 \mathrm{ps}$ time constant derived indirectly from the magnetic field dependence of time integrated escape efficiencies for the spin-allowed backward ET process in $\mathrm{MB}^{*} \cdots \mathrm{Fc}^{+}$radical pairs [6] This supports the notion that the observed time constant of 1 ps is an ET time.

In the following the time constants for FET and REC are treated in the framework of non-adiabatic ET theory (see e.g. refs. $[26,27]$ ) in its simplest form. In this theory the ET rate constant $k_{\mathrm{ET}}$ is given by

$k_{\mathrm{ET}}=\frac{2 \pi V^{2}}{(4 \pi \lambda k T)^{1 / 2} \hbar} \exp \left[-\frac{(\lambda+\Delta G)^{2}}{4 \lambda k T}\right]$,

where $V$ is the electronic coupling and $\lambda$ the total reorganization energy for the ET process. Under the assumption that $V$ and $\lambda$ are equal for FET and REC these two parameters can be determined from the observed rate constants provided that the respective free enthalpy changes $\Delta G$ are known. These free energy changes can be calculated from electrochemical and spectroscopic data using Eq. (5) for FET and Eq. (6) for REC.

$$
\begin{aligned}
& \Delta G_{\mathrm{fet}}=-E_{\mathrm{s}}-e\left(E_{\mathrm{MB}^{+} / \mathrm{MB}^{-}}-E_{\mathrm{Fc}^{+} / \mathrm{Fc}}\right), \\
& \Delta G_{\mathrm{rec}}=e\left(E_{\mathrm{MB}^{+} / \mathrm{MB}^{-}}-E_{\mathrm{Fc}^{+} / \mathrm{Fc}}\right) .
\end{aligned}
$$

The value for the singlet energy $E_{\mathrm{s}}$ of $\mathrm{MB}^{+}$is 1.84 $\mathrm{eV}$ [7], the reduction potential $E_{\mathrm{MB}^{+}} \mathrm{MB}^{-}$of $\mathrm{MB}^{+}$in acetonitrile is $-390 \mathrm{mV}$ (SCE) [28] and the reduction potential $E_{\mathrm{Fc}^{+} / \mathrm{Fc}}$ of ferrocene in acetonitrile is $310 \mathrm{mV}$ (SCE) [12], which is taken to be similar to that of FcN (see Section 2). Based on these values 
the resulting free enthalpy changes are $-1.15 \mathrm{eV}$ for $\Delta G_{\mathrm{fet}}$ and $-0.7 \mathrm{eV}$ for $\Delta G_{\mathrm{rec}}$. However, these energetic values have to be treated with caution since they refer to equilibrium conditions which might or might not hold for the ultrashort time regime of the processes under study here.

The ET rate constants $k_{\mathrm{ET}}$ are $1 /(380 \mathrm{fs})$ for the FET and $1 /(1 \mathrm{ps})$ for the REC. Using these values Eq. (4) yields $1.04 \mathrm{eV}$ for the reorganization energy $\lambda$ and $0.013 \mathrm{eV}$ or $100 \mathrm{~cm}^{-1}$ for the electronic coupling $V$. Relying on just two values - one for the FET and one for the REC - the parameters derived here have to be regarded as a crude estimate and are only discussed here to show that their magnitude is consistent with the ET mechanism postulated. The value of $\lambda$ of $\approx 1 \mathrm{eV}$ is reasonable, as, for example, the determined reorganization energy of the ferrocene /ferrocenium self-exchange reaction in acetonitrile is $1.06 \mathrm{eV}$ [29]. The electronic coupling $V \approx$ $100 \mathrm{~cm}^{-1}$ compares well with $V \approx 120 \mathrm{~cm}^{-1}$ obtained for a similar intermolecular D/A system for close-contact ET: the aniline/oxazine 1 [30], oxazine 1 being the oxa-analogue of methylene blue.

\subsubsection{Slow components of REC}

An interesting feature of the ground state recovery kinetics in the presence of the highest FcN concentrations is still to be discussed. In contrast to the behaviour of the ${ }^{1} \mathrm{MB}^{+*}$ state which is completely depleted after less than 5 ps (Fig. 3) the ground state recovery shows minor decay components with time constants of $9.2(11 \%)$ and $60 \mathrm{ps}(9.9 \%)$ and furthermore includes a long-lived amplitude ('offset') of $5.4 \%$ which indicates some escape from the geminate RPs to free radicals. According to the spin chemical model, the geminate REC of the singletphased RP is superimposed by the $S \rightleftharpoons T$ equilibration process of the RP. Since the triplet-phased RP cannot recombine directly the overall recombination rate of the RP will decrease concomitant with its singlet character. If diffusional separation were negligible one would expect the overall recombination kinetics to slow down to a rate constant of about $1 / 4$ of that of a pure singlet RP after a time of the order of the spin relaxation time. Inspection of the ground state recovery at high $\mathrm{FcN}$ concentration (Fig. 4) reveals that the fast decay tends to slow down after about $2-3$ ps. Such a behaviour is in fact consistent with a situation where the spin relaxation time is several times longer than the time constant of REC of pure singlet RPs, which is in qualitative accord with the conclusions from the spin chemical investigation in Ref. [6] yielding $k_{\text {bet }}=1 /(1.6 \mathrm{ps})$ and $\tau_{\mathrm{s}}=6 \mathrm{ps}$ for the MB $\cdots \mathrm{Fc}^{+} \mathrm{RPs}$ which may be expected to behave similar to the $\mathrm{MB} \cdot \cdots \mathrm{FcN}^{+}$ RPs investigated in the present work. Our reasoning here is qualitative and will have to be substantiated by detailed quantitative simulations including spin evolution as well as diffusional separation. However, it can be maintained that on this qualitative level the present findings based on direct time-resolved observations are fully consistent with the more indirect conclusions from the previous spin chemical investigation.

\section{Conclusions}

The phenomenology of forward electron transfer and recombination in the donor/acceptor system dimethylaminomethylferrocene/methylene blue has been studied in pump probe experiments on the fs to ps timescale. At high donor concentration the major kinetic components exhibit the following features.

(1) The forward electron transfer from the ferrocene component to methylene blue singlet (390 fs) is at least twice as fast as the corresponding recombination process ( $1 \mathrm{ps}$ ).

(2) The 1 ps recombination time is several times shorter than the spin relaxation time of the radical pair which should be governed by the paramagnetic relaxation mechanism in the ferricenium moiety. The result concerning the order of magnitude and the relative ordering of rate constants of ET and spin relaxation is fully consistent with the previous analysis of the effects of large magnetic fields on time-integrated free radical yields for the closely related ferrocene/methylene blue donor/acceptor system.

These results are encouraging for further investigations aiming for a direct ps time-resolved detection of the modulation of geminate recombination kinetics by high magnetic fields. Employing systems with chemically linked donor and acceptor components wherein diffusive separation of the radical pairs is suppressed will prove particularly interesting and will make a full theoretical simulation more feasible. 
Corresponding fs time-resolved experiments with linked systems of the ferrocene/methylene blue type in fields up to $9 \mathrm{~T}$ are in progress right now in the Munich group.

These experiments in high magnetic fields carry the potential to develop into a unique experimental tool for disentangling complex reaction patterns in transition metal redox systems and to identify recombination processes in ultrafast rise and decay patterns which are otherwise hardly accessible to interpretation.

\section{Acknowledgements}

We thank Professor J. Jortner for stimulating discussions. PG is indebted to the Fonds der Chemische Industrie for a scholarship (Kekulé-Stipendium). Financial support of the Deutsche Forschungsgemeinschaft (SFB 377) and the Volkswagenstiftung is gratefully acknowledged.

\section{References}

[1] D. BürBner, H.J. Wolff, U.E. Steiner, Angew. Chem. Int. Ed. Engl. 33 (1994) 1772.

[2] W. Lersch, M.E. Michel-Beyerle, Chem. Phys. 78 (1983) 115.

[3] E.L. Frankevich, A.I. Pristupa, V.I. Lesin, Chem, Phys. Lett. 47 (1977) 304

[4] M. Goez, in: Dynamics during Spectrosopic Transitions, E. Lippert, J.D. Macomber, (Eds.), Springer-Verlag, Berlin, 1995, p. 305. Recent review on CIDNP and CIDEP.

[5] G. Biesser, Ph.D. thesis, Technische Universität München (1996).

[6] P. Gilch, M. Linsenmann, W. Haas, U.E. Steiner, Chem. Phys. Lett. 254 (1996) 384

[7] M. Kojima, Y. Kuriyama, H. Sakuragi, K. Tokumaru, Bull. Chem. Soc. Jpn. 64 (1991) 2724.

[8] L. Manring, J. Eriksen, C. Foote, J. Am. Chem. Soc. 102 (1980) 4275 .
[9] M. Nemoto, H. Kokubun, M. Koizumi, Bull. Chem. Soc. Jpn. 42 (1969) 1223.

[10] S. Tamura, K. Kikuchi, H. Kokubun, A. Weller, Z. Phys Chem. NF 121 (1980) 165.

[11] C. Barraqué, J. Vedel, B. Trémillon, Bull. Soc. Chim. Fr. (1968) 3421.

[12] H. Henning, O. Guirtler, J. Organometal. Chem. 11 (1968) 307.

[13] A.A. Pendin, P.K. Leont'evskaya, T.I. L'vova, B.P Nikol'skii, Dokl. Akad. Nauk SSSR 189 (1969) 115.

[14] H.-M. Koepp, H. Wendt, H. Strehlow, Z. Elektrochem. 64 (1960) 483

[15] F. Pöllinger, C. Musewald, H. Heitele, M.E. Michel-Beyerle, C. Anders, M. Futscher, G. Voit, H. Staab, Ber. Bunsenges. Phys. Chem. 100 (1996) 2076.

[16] H. Lossau, A. Kummer, R. Heinecke, F. Pöllinger-Dammer C. Kompa, G. Bieser, T. Jonsson, C.M. Silva, M.M. Yang, D.C. Youvan, M.E. Michel-Beyerle, Chem. Phys. 213 (1996) l.

[17] F. Salin, F. Estable, F. Saviot, in: Tunable Femtosecond Sources and Optical Parametric Generators, P.F. Barbara, W.H. Knox, G.A. Mourou, A.H. Zewail, (Eds.), SpringerVerlag, Berlin, 1994, p. 194.

[18] K. Weidemaier, H.L. Tavernier, S.F. Swallen, M.D. Fayer, J. Phys. Chem. A 101 (1997) 1887.

[19] M. Enescu, L. Krim, L. Lindqvist, W. Tieqiang, J. Photochem. Photobiol. B: Biol. 22 (1994) 165.

[20] Y.S. Sohn, D.N. Hendrickson, H.B. Gray, J. Am. Chem. Soc 93 (1970) 3603

[21] K. Kikuchi, S. Tamura, C. Iwanaga, H. Kokubun, Z. Phys. Chem. NF 106 (1977) 17.

[22] M.L. Horng, J.A. Gardecki, A. Papazyan, M. Maroncelli, J. Phys. Chem. 99 (1995) 17311.

[23] M.L. Ceccarani, P. Sassi, R.S. Cataliotti, J. Chem. Soc Faraday Trans. 90 (1994) 1397

[24] J.D. Dunitz, L.E. Orgel, A. Rich, Acta Crystallogr. 9 (1956) 373.

[25] S.L. Murov, I. Carmichael, G.L. Hug, Handbook of Photochemistry, (Eds.), Marcel Dekker, New York, 1993.

[26] J. Jortner, J. Chem. Phys. 64 (1976) 4860

[27] R.A. Marcus, J. Chem. Phys. 81 (1984) 4494.

[28] K. Golnick, Ann. NY Acad. Sci. 171 (1970) 89.

[29] R.M. Nielson, G.E. McManis, L.K. Safford, M.J. Weaver, J Phys. Chem. 93 (1989) 2152.

[30] Y. Nagasawa, A.P. Yartsev, K. Tominaga, A.E. Johnson, K Yoshihara, J. Chem. Phys. 101 (1994) 5717. 